\title{
Loose ENDs: Electronic Nicotine Delivery Systems and the FDA's Recent Enforcement Policy
}

Authors:

Disclosure:

Acknowledgements:

Received:

Accepted:

Keywords:

Citation:
Saira Ahmad, M. Flori Sassano, *Robert Tarran

Department of Cell Biology \& Physiology, University of North Carolina,

Chapel Hill, North Carolina, USA

*Correspondence to robert_tarran@med.unc.edu

The authors have declared no conflicts of interest.

This work was funded by NIH/NHLBI HL135642 and NIH/NHLBI/FDA HL153698.

24.06 .20

02.09 .20

Airway, electronic cigarette (e-cigarette), lung, nicotine, vaping.

EMJ Respir. 2020;8[1]:93-96.

\section{INTRODUCTION}

Electronic nicotine delivery systems (ENDS), including electronic cigarettes (e-cigarettes), have been commercially available since the early 2000s. Since then, their use has increased both among adults and adolescents. ${ }^{1}$ E-cigarettes consist of an electric heater that aerosolises a liquid (e-liquid), which usually contains nicotine dissolved in a liquid vehicle (propylene glycol [PG] and vegetable glycerin [VG]) and chemical flavours. ${ }^{2}$ These flavours served to mask the bitter taste of nicotine, and they also facilitate the initiation and uptake of e-cigarettes by attracting youth and young adults. ${ }^{3}$ In the USA alone, there are over 1,200 different vendors and over 8,000 flavors. ${ }^{4} \mathrm{E}$-cigarettes have undergone a series of design evolutions and are currently sold both as tank-based devices (e.g., 'mods', so called because of their customisability) and as cartridge-based (JUUL-type) devices, with cartridge-based e-cigarette products currently being the most popular form of e-cigarette in the USA.5,6 These products are especially popular among young adults and adolescents, ${ }^{7}$ and may facilitate nicotine addiction and dependence. ${ }^{8}$

The long-term impact of vaping on the cardiovascular system, and the cancer risk, remain to be determined. However, increasing evidence shows that exposure to e-cigarette vapor in human models affects pulmonary and vascular functions, ${ }^{9,10}$ although the impact of individual flavours to these effects remain to be fully determined. More recently, e-cigarette or vaping product use associated lung injury (EVALI) has been described, which was particularly prevalent in youth, and has further raised concern over e-cigarette safety." In many cases, EVALI was most likely caused by the inclusion of vitamin E acetate, which was present to solubilise tetrahydrocannabinol, ${ }^{12}$ although not all EVALI patients used tetrahydrocannabinol, suggesting that other, as-yet-unknown, e-cigarette components may have been responsible." The EVALI outbreak of 2019 reconfirms the need for appropriate e-liquid regulation. As a case in point, the European Union's (EU) Tobacco Product Directive banned both the use of refillable cartridges and the inclusion of vitamins in e-liquids, ${ }^{13}$ and to date, EVALI has essentially been absent from the EU, ${ }^{14}$ suggesting that appropriate e-cigarette legislation can influence health outcomes.

The Family Smoking Prevention and Tobacco Control Act of 2009 banned the use of all natural and artificial flavour compounds (except for menthol) in combustible cigarettes and any of their component parts to eliminate flavoured 
tobacco products that held special appeal in the youth market. However, this ruling did not apply to other tobacco products, such as 'little cigars', and also did not apply to e-cigarettes. In 2020, the U.S. Food and Drug Administration (FDA) finalised their enforcement policy on flavoured cartridge-based e-cigarette products, including fruit and mint flavors. ${ }^{15}$ In this commentary, the authors discuss the potential impact of flavours on human health and addiction, and the implications on regulation.

\section{TYPES OF FLAVOURS}

Flavour is most commonly sensed by taste receptors in the mouth and the five flavours bitter, salty, sour, sweet, and umami (savoury), can be sensed. These receptors are typically either G-protein coupled receptors (e.g., taste receptor TAS1R2, sweet) or ion channels (e.g., the epithelial $\mathrm{Na}^{+}$channel, salty). ${ }^{16,17}$ Flavour, and activation of taste receptors, is an important part of the smoking/vaping experience. For example, the inclusion of sweet flavours is reinforcing and has been shown to potentiate the effect of nicotine on the brain. ${ }^{18}$

\section{E-liquids contain many different flavours,} including aldehydes (vanillin, benzaldehyde, cinnamaldehyde, damascenone), benzyl alcohol, terpenes (linalool, limonene, farnesol), pyrazines, menthol, and sweet flavours including ethyl maltol. These flavours are mixed to produce the thousands of commercially available e-liquids. Many of these flavours have also been used in the food and perfumes/cosmetics industries. However, their safety in the lung, at levels inhaled by e-cigarette users, is uncertain because the majority of toxicology studies for these flavours were carried out following oral ingestion only. ${ }^{19}$ For example, high doses of inhaled diacetyl (2,3 butanedione, used for buttery flavour in popcorn) can lead to severe lung disease, in the form of bronchiolitis obliterans (aka 'popcorn lung'). ${ }^{20}$ Despite the danger, diacetyl has been detected in some e-liquids. ${ }^{21,22}$

Flavoured e-liquids and individual flavours have been shown to induce toxicity and/or exert biological effects, which has been reviewed elsewhere. ${ }^{23}$ The number of flavours used in an e-liquid varies from product to product; previously, the authors identified 100 different flavours in 148 e-liquids, and found that vanillin was the most common flavour. ${ }^{22}$ Interestingly, the number of flavours contained within an e-liquid directly correlated with degree of toxicity. ${ }^{22}$ Flavour concentrations in most e-liquids have not yet been determined; however, both vanillin and cinnamaldehyde were typically in the $\mathrm{mmol} / \mathrm{L}$ range, and in some cases, cinnamaldehyde levels exceeded $1 \mathrm{~mol} / \mathrm{L}^{22,24}$ Flavourings such as vanillin and cinnamaldehyde are aldehydes that have the potential to form adducts with proteins and DNA. This adduct binding can alter protein function and possibly cause DNA mutations as DNA adduct levels have been considered to serve as biomarkers for carcinogen exposure. . $3,25,26$

\section{ROLE OF FLAVOURS IN UPTAKE/ APPEAL TO YOUTH}

Most people who start using nicotine do so as a teenager. $^{27,28}$ For cigarettes, the use of flavours to mask the unpleasant taste (bitter) and irritation of the combusted cigarette has been well described. ${ }^{29-31}$ Moreover, menthol directly activates transient receptor potential channels in pulmonary neurons to suppress cough and irritation, thus making it easier to overcome the initially unpleasant effects of tobacco smoke inhalation. 32 The 2019 National Youth Tobacco Survey (NYTS) found that over 5 million middle and high school students in the USA (10.5\% and $27.5 \%$, respectively) were e-cigarette users, defined as having used e-cigarettes within the last 30 days, ${ }^{33}$ the majority of whom used cartridgebased products. In addition to masking the unpleasant sensations of nicotine, e-cigarettes can be made in appealing 'candy', 'dessert', and 'fruit' flavours, among several others, which also aids with marketing and appeal to teenagers. ${ }^{34-36}$

\section{UPDATE ON RECENT LEGISLATION}

Having a broad range of commercially-available flavours is a common marketing practice used by e-liquid vendors. ${ }^{37}$ The FDA has taken steps to regulate e-cigarette sales in order to prevent sales to youth. First, they deemed e-cigarettes to be tobacco products and included them in the 2009's Family Smoking Prevention and Tobacco Control Act. ${ }^{38}$ They subsequently issued an "Advanced Notice of Proposed Rulemaking" that gives importance to the regulation of flavours in 
tobacco products. ${ }^{39}$ This year, the FDA finalised their rules on flavours and have banned flavoured, cartridge-based e-cigarettes. ${ }^{40}$ Given that JUULtype cartridges have the largest market share, this is certainly a step in the right direction. More specifically, the FDA has banned: a) any flavoured, cartridge-based ENDS product (other than a tobacco- or menthol-flavoured ENDS product); b) all other ENDS products for which the manufacturer has failed to take (or is failing to take) adequate measures to prevent minors' access; and c) any ENDS product that is targeted to minors or likely to promote use of ENDS by minors. ${ }^{40}$

This legislation is certainly a step in the right direction. For example, JUUL previously sold eight different flavours, including Crème brûlée, mint, fruit medley, and mango, with mint, mango, and fruit medley being the most popular amongst school-age vapers. ${ }^{41}$ However, now they only sell 'Classic Tobacco', 'Virginia Tobacco', and 'Menthol', which will likely limit appeal to youth, as intended by the FDA. Unfortunately, this legislation has some loopholes. For example, while sales of e-cigarette cartridges containing flavoured e-liquids have been banned, individual containers of flavoured e-liquids, including those with fruit, candy, and other enigmatic names are still commercially available via a number of websites. Moreover, it is now possible to purchase offmarket, empty/refillable cartridges for cartridgestype devices. Therefore, at the moment it is relatively easy to circumvent the flavour ban by purchasing flavoured e-liquids, and putting these into either second- or third-generation e-cigarette devices which are refillable, or by using refillable cartridges for JUUL-type e-cigarettes. One might argue that these attractively-named, flavoured e-liquids are targeted at minors, in which case, the FDA needs to enforce the sale of flavoured non-cartridge e-liquids in order to fully enforce the flavour ban for cartridge-based e-cigarettes.

Another important omission from the FDA's rule is the continued sale of menthol. As mentioned above, menthol directly stimulates neuronal transient receptor potential channels to suppress cough after smoke inhalation and is arguably the most biologically active of the available flavours. ${ }^{32}$ There is strong evidence that menthol initiates smoking, and menthol is popular among young smokers. Indeed, cigarette and e-cigarette company marketing strategies have been based around this information. Thus, given its impact on smoking initiation, it is hard to explain why menthol continues to be available despite the flavour ban. The tobacco industry continues to lobby for the promotion of their products and USA states with more active tobacco lobbies are less likely to legislate for tobacco control. ${ }^{42}$ However, the degree to which lobbying has influenced the continued availability of menthol remains to be determined.

\section{CONCLUSIONS}

In conclusion, the FDA's recent ban on flavoured e-cigarette cartridges is an important milestone in the regulation of this relatively new product. Given that it took many individuals, several decades, and innumerable lawsuits before conventional tobacco was regulated, by comparison the FDA has moved at "light speed" with their deeming rules and their flavour ban. However, loose ends need to be addressed, including the availability of e-liquid flavours in other forms, the availability of refillable cartridges, and the lack of regulation surrounding menthol.

\section{References}

1. Bals R et al. Electronic cigarettes: a task force report from the European Respiratory Society. Eur Respir J. 2019;53(2):1801151.

2. Hahn J et al. Electronic cigarettes: overview of chemical composition and exposure estimation. Tob Induc Dis. $2014 ; 12(1): 23$.

3. Leventhal AM et al. Flavored e-cigarette use and progression of vaping in adolescents. Pediatrics. 2019;144(5):e20190789.

4. U.S. Department of Health and Human Services, E-Cigarette Use Among Youth and Young Adults. A Report of the Surgeon General (2016), Atlanta, U.S. Department of Health and Human Services, Centers for Disease Control and Prevention, National Center for Chronic Disease Prevention and Health Promotion, Office on Smoking and Health.
5. Fadus $M C$ et al. The rise of e-cigarettes, pod mod devices, and JUUL among youth: factors influencing use, health implications, and downstream effects. Drug Alcohol Depend. 2019;201:85-93.

6. Huang $\mathrm{J}$ et al. Vaping versus JUULing: how the extraordinary growth and marketing of JUUL transformed the US retail e-cigarette market. Tob Control. 2019;28(2):146-51. 
7. Willett JG et al. Recognition, use and perceptions of JUUL among youth and young adults. Tob Control. 2019;28(1):115-6

8. Thorndike AN. E-Cigarette use by young adult nonsmokers: nextgeneration nicotine dependence? Ann Intern Med. 2019;170(1):70-1.

9. Bozier J et al. The evolving landscape of electronic cigarettes: a systematic review of recent evidence. Chest. 2020;157(5):1362-90.

10. Gotts JE et al. What are the respiratory effects of e-cigarettes? BMJ. 2019;366:15275.

11. Crotty Alexander LE et al. NIH Workshop Report: e-cigarette or vaping product use associated lung injury (EVALI): developing a research agenda. Am J Respir Crit Care Med. 2O2O;DOI: 10.1164/rccm.2019122332WS.

12. Blount BC et al. Vitamin $E$ acetate in bronchoalveolar-lavage fluid associated with EVALI. N Engl J Med. 2020;382(8):697-705.

13. European Commission. Questions \& Answers: New rules for tobacco products. 2014. Available at: https://ec.europa.eu/commission/ presscorner/detail/en/MEMO_14_134. Last accessed: 4 September $\overline{2} \mathrm{O} 2 \overline{\mathrm{O}}$.

14. Blagev DP. No man is an island: e-cigarette, or vaping, associated lung injury in Europe. Eur Respir J. 2020;55(2):1902419.

15. U.S. Food and Drug Administration (FDA). Enforcement priorities for electronic nicotine delivery system (ENDS) and other deemed products on the market without premarket authorization center for tobacco products. 2020. Available at: https:// www.fda.gov/regulatory-information/ search-fda-guidance-documents/ enforcement-priorities-electronicnicotine-delivery-system-ends-andother-deemed-products-market. Last accessed: 4 September 2020.

16. Oka $Y$ et al. High salt recruits aversive taste pathways. Nature. 2013;494(7438):472-5.

17. Treesukosol $Y$ et al. The functional role of the T1R family of receptors in sweet taste and feeding. Physiol Behav. 2011;105(1):14-26.

18. Kroemer NB et al. Sweet taste potentiates the reinforcing effects of e-cigarettes. Eur Neuropsychopharmacol. 2018:28(10):1089-102

19. Klager $\mathrm{S}$ et al. Flavoring chemicals and aldehydes in e-cigarette emissions. Environ Sci Technol.
2017:51(18):10806-13.

20. Kreiss K. Flavoring-related bronchiolitis obliterans. Curr Opin Allergy Clin Immunol. 2007;7(2):162-7.

21. Farsalinos KE et al. Evaluation of electronic cigarette liquids and aerosol for the presence of selected inhalation toxins. Nicotine Tob Res. 2015;17(2):168-74.

22. Sassano MF et al. Evaluation of e-liquid toxicity using an open-source high-throughput screening assay. PLoS Biol. 2018;16(3):e2003904.

23. Kaur $\mathrm{G}$ et al. Mechanisms of toxicity and biomarkers of flavoring and flavor enhancing chemicals in emerging tobacco and non-tobacco products. Toxicol Lett. 2018; 288:143-55.

24. Clapp PW et al. Cinnamaldehyde in flavored e-cigarette liquids temporarily suppresses bronchial epithelial cell ciliary motility by dysregulation of mitochondrial function. Am J Physiol Lung Cell Mol Physiol. 2019;316(3):L470-86.

25. Erythropel $\mathrm{HC}$ et al. Formation of flavorant-propylene glycol adducts with novel toxicological properties in chemically unstable e-cigarette liquids. Nicotine Tob Res. 2019;21(9):1248-58.

26. Wiencke JK. DNA adduct burden and tobacco carcinogenesis. Oncogene. 2002;21(48):7376-91.

27. Chadi $\mathrm{N}$ et al. Understanding the implications of the "vaping epidemic" among adolescents and young adults: a call for action. Subst Abus. 2019;40(1):7-10.

28. Cullen KA et al. Notes from the field: use of electronic cigarettes and any tobacco product among middle and high school students - United States, 2011-2018. MMWR Morb Mortal Wkly Rep. 2018;67(45):1276-7.

29. Mead EL et al. E-cigarette palatability in smokers as a function of flavorings, nicotine content and propylthiouracil (PROP) taster phenotype. Addict Behav, 2019:91:37-44.

30. Pullicin AJ et al. Impacts of nicotine and flavoring on the sensory perception of e-cigarette aerosol. Nicotine Tob Res. 2020;22(5):806-13.

31. Wickham RJ. The biological impact of menthol on tobacco dependence. Nicotine Tob Res. 2019;ntz239. [Epub ahead of print].

32. Willis DN et al. Menthol attenuates respiratory irritation responses to multiple cigarette smoke irritants.
FASEB J. 2011;25(12):4434-44.

33. Cullen KA et al. E-Cigarette use among youth in the United States, 2019. JAMA. 2019;322(21):2095-103.

34. Cullen KA et al. Flavored tobacco product use among middle and high school students - United States, 20142018. MMWR Morb Mortal Wkly Rep. 2019;68(39):839-44

35. Leventhal AM et al. Effects of non-tobacco flavors and nicotine on e-cigarette product appeal among young adult never, former, and current smokers. Drug Alcohol Depend. 2019;203:99-106.

36. Villanti $A C$ et al. Flavored tobacco product use in youth and adults: findings from the first wave of the PATH study (2013-2014). Am J Prev Med. 2017;53(2):139-51.

37. Garrison KA et al. A fMRI study on the impact of advertising for flavored e-cigarettes on susceptible young adults. Drug Alcohol Depend 2018:186:233-41.

38. Food and Drug Administration, HHS Deeming tobacco products to be subject to the Federal Food, Drug, and Cosmetic Act, as amended by the Family Smoking Prevention and Tobacco Control Act; restrictions on the sale and distribution of tobacco products and required warning statements for tobacco products. Final rule. Fed Regist. 2016;81(90):28973-9106.

39. Food and Drug Administration, HHS. Regulation of flavors in tobacco products. 2018. Available at: https://www.federalregister.gov/ documents/2018/03/21/2018-05655/ regulation-of-flavors-in-tobaccoproducts. Last accessed: 4 September 2020.

40. U.S. Food and Drug Administration (FDA). FDA finalizes enforcement policy on unauthorized flavored cartridge-based e-cigarettes that appeal to children, including fruit and mint. 2020. Available at: https://www.fda.gov/news-events/ press-announcements/fda-finalizesenforcement-policy-unauthorizedflavored-cartridge-based-ecigarettes-appeal-children. Last accessed: 7 September 2020.

41. Leventhal AM et al. Flavors of e-cigarettes used by youths in the United States. JAMA. 2019; 322(21):2132-4

42. Maclean JC et al. What factors predict the passage of state-level e-cigarette regulations? Health Econ. 2018;27(5):897-907. 